\title{
BASIC ANALYSIS OF BIPED ROBOT LEGS
}

\author{
Harshawardhan Kumar Unsasi \\ Department of Mechanical Engineering \\ College of Engineering, Anna University, Chennai, \\ Tamil Nadu, India.
}

\begin{abstract}
The basic but fundamental component of biped robots, mechanical legs have a high versatility and manoeuvrability; these capabilities of the legs help robots to be highly agile in an extremely cluttered environment. A press release from IFR (International Federation of Robotics) et al. (2018) says that Global industrial sales of robots have a $+\mathbf{1 4 \%}$ increase on average per year, this pushes the study and research on robot to next level. A large number of robotic institutions and researchers around the world have studied robots with mechanical legs. In this article, information from many items is collected and explained in detail about the design and advantages of a biped robot. This article also discusses the stability control and motion control of the robot elaborately. The motivation of this article is to provide a clear understanding of mechanical legs in biped robots and help students and engineers to design a more efficient robotic leg.
\end{abstract}

Keywords - Degree of Freedom, Biped robot, Support Polygon, Zero Moment Point, Foot Rotation Indicator, Centre of Pressure

\section{INTRODUCTION}

Ever since robots have created, humans have always dreamt of human-like robots with legs and hands. Legged robots have received much attention in the last few years. Today's most advanced robots like "ASIMO - a humanoid robot created by Honda in 2000", "Atlas - a bipedal humanoid robot developed by Boston Dynamics", "Nao - programmable humanoid robot developed by Aldebaran Robotics" can tramp along flat and climb along inclined surfaces, climb up and downstairs, and slog through rough terrain. Some of many robots can even jump. These robots require a high level of stability and control for its locomotion. A small area of contact by the feet of the robot on the ground increases the complexity of the design of the biped robot.

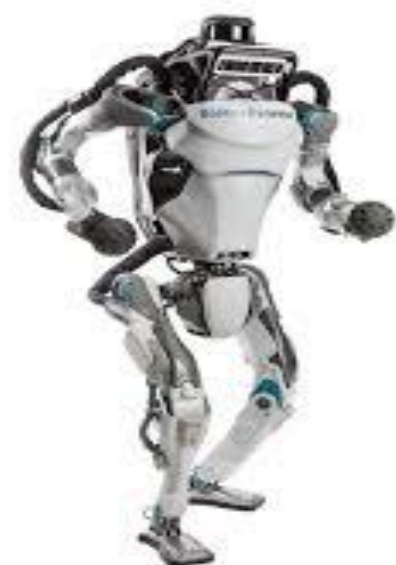

Fig 1. Atlas Robot

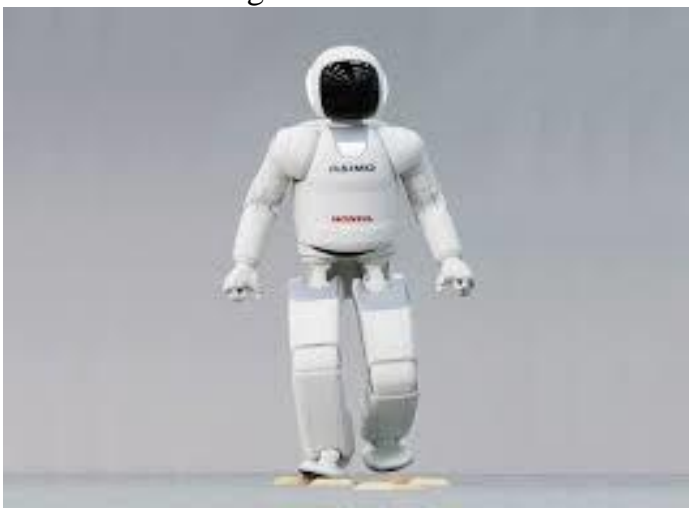

Fig 2. ASIMO Robot

The development of a bipedal walking system is a multidisciplinary subject; it includes mechanics, electricals, electronics, computer science and much more field of studies.

\section{MECHANICAL DESIGN OF A BIPED ROBOT}

There is various design consideration while designing a robot. Some of the significant factors considered are,
A. Size of the Robot
B. Degree of freedom
C. Actuator
D. Load and stresses at joints

\section{A. Size of the Robot-}

When a robot is designed, the main factor which affects the overall weight of the robot is the size of the robot. The size of 


\section{International Journal of Engineering Applied Sciences and Technology, 2019 \\ Vol. 4, Issue 8, ISSN No. 2455-2143, Pages 177-179 \\ Published Online December 2019 in IJEAST (http://www.ijeast.com)}

the robot is affected by various factors. When the size of the robot is different, there are differences in the power of actuators (servo motors) for driving a joint of the robot legs. A small, light-weight and high strength, optimum servo motors should be designed for this purpose. When the size of the robot is different, there are differences in the optimum frame structure of the robot(skeleton). So, proper frame structure should also be selected for this purpose. Manufacturing technology is essential in the development of the robot. If intricate shapes and size of the robot is selected, it should be checked appropriately with the manufacturing technology for fruitful implementations.

As the body structure of humans mainly inspires biped robots, the size and weight of modern robots are compared and designed according to human measurements.

\section{B. Degree of Freedom -}

Human legs are omnidirectional, and they have six degrees of freedom. They are, Hip- 3 DOF, Knee- 1 DOF, Ankle- 2 DOF). So, it is crucial to design a robotic leg as compared to human limbs. That means the robot must be physically capable of changing walking directions, walk up and downstairs, and other similar tasks. For performing all the above functions mentioned, robots should have a sufficient degree of freedoms. If a robot can move only in one direction, say forward and backwards, that would make the robot difficult to change its directions, move sideways and climb inclined surface and stairs. So, a robot needs to have a sufficient degree of freedom.

\section{Actuator}

The component which is responsible for the actual motion of the robot are actuators. It is highly essential for a humanoid robot to mimic the human body, so actuators with high power but less weight is used as muscles and joints for the robot.

Selecting an actuator for the robot is a vital task. Power to weight ratio is the critical parameter used to determine the appropriate actuator for the leg. After knowing the size and weights for the robot, a suitable actuator could then be chosen. The most common types of actuators are the hydraulic, piezoelectric, pneumatic, ultrasonic and electric actuators. Hydraulic actuators have good power to weight ratios, but they are not light-weight. Pneumatic actuators are lightweight, but they do not have high power-weight proportions. Both hydraulic and pneumatic actuators require a bulky compressor and pump, which cannot be fitted for a walking robot. Piezoelectric actuators work by the piezoelectric effect. These actuators can be used for exact positioning conditions, and it can handle very high pressures in static as well as dynamic conditions. Ultrasonic actuators are designed to supply micrometre movements, and that they are utilized in dominant vibrations and fast switch functions. The sole effective and economical choice is electrical motors. Coreless motors are preferred for low speed and high load applications.
For selecting actuators, torque and speed of all the joint should be calculated systematically and analyzed. Generally, the knee and hip pitch require the highest torque. For note, the maximum torque and speed points do not occur at the same time.

\section{Load and Stresses at Joints}

From comparison with the human body, it is estimated that pressures on the hip and knee of the human body can reach up to 4 to 7 times the body weight, respectively. The load of the robot must be uniformly distributed between both legs for better and stable motion of the robot. For an example, if the estimated weight of the biped robot is $60 \mathrm{Kg}$, and with the load factor of 5.5(average of 4 and 7), the load on the hip would be $3237.3 \mathrm{~N}$ (taking $\mathrm{g}=9.81 \mathrm{~m} / \mathrm{s} . \mathrm{s}$ ). And as of knee is taken into account, the load of $3237.3 \mathrm{~N}$ is equally distributed between both legs. All the weights should be taken into account when estimating the size and material for the hip and knee joints.

The impact on the legs can be reduced by using shockabsorbing materials. In some of the robots, the shockabsorbing equipment is fitted in the foot. A new "programmable viscoelastic materials" designed by MIT team, Massachusetts can also be used as material for the robot. Viscoelastic material has a customizable level of stiffness and elasticity, which allows the designer to select the stiffness of equipment for different application and terrains.

\section{StABILITY AND Motion CONTROL}

The critical aspect of the stability of the robot is the postural balance of biped systems respective to the shape and size of the feet of the robot. Convex hull of the foot support area of the legs is called support polygon, and it contributes a significant role in the stability of the robot.

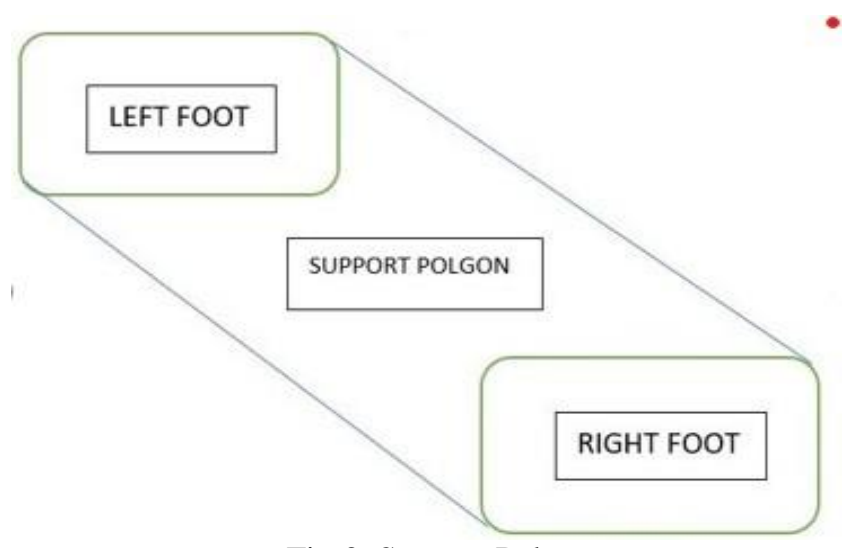

Fig 3. Support Polygon

For robots with non-zero support polygon, the stability is generally analyzed by Zero-Moment-Point (ZMP). It is defined as the position on which the net moment of the inertial forces and the forces due to gravity have zero components 


\section{International Journal of Engineering Applied Sciences and Technology, 2019 \\ Vol. 4, Issue 8, ISSN No. 2455-2143, Pages 177-179 \\ Published Online December 2019 in IJEAST (http://www.ijeast.com)}

along the straight axis. The robot needs to have ZMP within the support polygon for efficient and stable movement.

Centre-of-pressure (CP) is another concept used to analyze the postural stability of the robot. CP is defined as the point where the resultant of the ground reactions force acts. If the robot is designed such that the ZMP lies inside the support polygon, then ZMP will coincide with CP. If ZMP moves outside the support polygon, the robot will lose the stability of motion. However, the degree of instability is not indicated by the ZMP criterion.

For the locomotion of biped robots, rotational equilibrium of the foot is one of the critical parameters for the postural stability of the biped robot. When the robot is stationary, the rotational balance of the feet is determined by the location of the centre of mass of the robot. But, when the robot is in motion, the rotational properties of the foot are decided by the position of the Foot Rotation Indicator (FRI) point. FRI is the point on the ground and foot contact surface, inside or outside the support polygon, where the resultant moment of the force or torque impresses on the foot is normal to the support. FRI point is the point on the foot ground contact surface where the net ground reaction should act to prevent foot rotation. The location of FRI point on foot represents or indicates the unbalanced force or torque on foot. More away, the FRI from the support polygon, more significant the unbalance moment which directly affect the stability of the robot.

Note, if the FRI remains within the support polygon, CP (Centre of pressure) $=$ ZMP (Zero moment point) $=$ FRI (Foot rotation indicator) and the supporting foot does not rotate.

\section{CONCLUSION}

The description of design steps in this paper provides a basic understanding of fundamental designing steps of the biped robot analogous to animals. The primary design considerations in the creation of biped robots are the size of the robot, DOFs, actuators, and stability and motion control of the robot.

\section{REFERENCE}

[1] Avery Thompson. New Shock-Absorbing Material Could Help Robots Take a Real Beating. Article in Popular Mechanics. Pp. 1-2, 2016.

[2] DW. Seward, A. Bradshaw, and F. Margrave. The anatomy of a humanoid robot. Robotica. Pp. 437-445.

[3] R.S Khurmi, J.K Ghupta. Theory of Machines. S. Chand publications. Pp 102-115.

[4] James J. Troy. Dynamic balance and walking control of biped mechanisms. Iowa State University Capstones, Theses and Dissertations. Pp. 9-11, 1995

[5] A. Unsworth. Lubrication of human joints. In Verna Wright and Eric Rad in, editors, Mechanics of Human
Joints: Physiology, Pathophysiology and Treatment. Pp. 137-162, 1993.

[6] K. Hirai, M. Hirose, Y. Haikawa and Takenaka. T. The development of honda humanoid robot. Pp. 1321-1327, 1998.

[7] Google images (www https://images.google.com/)

[8] Wikipedia (https://en.wikipedia.org/wiki/Humanoid_robot) 